duisent aussi du tétrachlorure de carbone $\mathrm{CGI}^{4}$, dont nous ávons parlé plus haut.

La production mondiale de la soude par l'électrolyse a été de 51000 tonnes en 1909 . Cette quantité représente le dixième de la production totale.

Oxygène et hydrogène. - Depuis 1897 , on a réalisé la préparation hydro-électrique de l'hydrogène et de l'oxygène, en électrolysant une solution de soude ou de potasse recouverte d'une couche d'huile pour empêcher l'acide carbonique de l'air de transformer les hydrates en carbonates

Il existe divers procédés : ceux de Garutti et de Schmidt travaillent en solution alcaline avec électrodes en fer, celui de Schoop en solution acide avec électrodes en plomb.

Le plus employé est le procédé Garutti, qui est exploité à Tivoli, à Lucerne, à Bruxelles et à Schiedam (Hollande). En France, il cst exploité à Saint-Vrain et par la Société l'Oxhydrique Française, avec des productions respectives de $400 \mathrm{~m}^{3}$ d'hydrogène et $200 \mathrm{~m}^{3}$ d'oxygène.

La Société Electro-Chimic fabrique aux Clavaux l'oxylithe et l'hydrolithe : ce sont des produits agglomérés en bloc qui jouissent de la propriété de dégager de l'oxygène ou 'de l'hydrogène lorsqu'on les immerge dans l'eau. Un kilogramme d'oxylithe fournit r 4 o litres d'oxygène et un kilogramme d'hydrolythe I ooo litres d'hydrogène.

Etant donné les nombreuses applications de l'oxygène et de l'hydrogène (gonflement des ballons, soudure autogène et découpage des métaux, approvisionnement des laboratoires et des pharmacies, elc.), cette industric aurait été susceplible de prendre un grand essor, mais les procédés do distillation fractionnée de l'air liquide (Linde et Claude) onl étć pour ello de redoutables et heureux concurrents et, en fait, la préparalion électrolytique de l'oxygène et de l'hydrogène a presque disparue.

Carborundum. - Le carborundum n'est autre chose que du: siliciure de carbone: Son extrême dureté, comparable à celle du diamant, le fait employer dans la fabrication des meules pour le polissage des métaux. On l'utilise aussi pour la construction des trottoirs et des marches non glissantes, où la cinculation est intense.

Enfin, il a trouvé un autre emploi intéressant dans le gạrnissage des fours métallurgiques, car il est insoluble dans le fer en fusion.

Aulres fabrications et applicalions diverses. - Les applications de l'électro-chimie s'étendent à un nombre extrếmement grand de produits, et des plus divers. M. Pinot n'a cité.que les principaux d'entre cux, mais voici, pour ter-miner, l'énumération d'un certain nombre des autres, ces fabrications étant d'importance fort inégale et souvent fort minime : Persulfate d'ammonium (Société d'Electro-Chimic dans son usine de Vallorbe). Chromate de sodium. Ferricyanure de potassium. Carbonate de plomb (céruse). Couleurs minérales (jaune do cadmium, bleu de Paris). Couleurs d'aniline (Badische Anilin und Soda Fabrik). Chloroforme et iodoforme. Produits de réduction de la mono-nitrobenzine. Siliciures alcalino-terreux. Scls de baryle. Phosphore (Maison Coignet, à Moutiers). Ozone.

On jugera, par cette revue empruntée dans une très large mesure au Rapport du distingué Secrétaire général de la Chambre syndicale des Forces hydrauliques, quel intérêt présente le remarquable travail de M. Robert Pinor.

(A suivre).

H.-L. Bernardin, Ingénieur des Arts et Manufactures.

\section{TRANSPORT D'ÉNERGIE}

\section{Protection des lignes aériennes contre les surtensions et les décharges atmosphériques (1)}

La protection des installations électriques contre les surtensions repose de nos jours sur des règles bien définics. Nous n'allons envisager, dans ce qui va suivre, que la question de la protection des lignes aériennes.

Nous avons défini ailleurs $\left({ }^{2}\right)$ ce que nous entendions par système complet de protection contre les surtensions dans les lignes aériennes à haute tension.

Comme on le voit sur la figure $x$, on peut dire que la protection n'est complète qu'aulant qu'il est prévu des dispositions de protection contre l'action des coups de foudre directs (A), des phénomènes atmosphériques à haute, moyennc ct basse fréquence ou à fréquence nulle $(B, C, D)$. Elle sera d'autant plus intéressante qu'on aura atteint ce but avec un nombre minimum d'appareils.

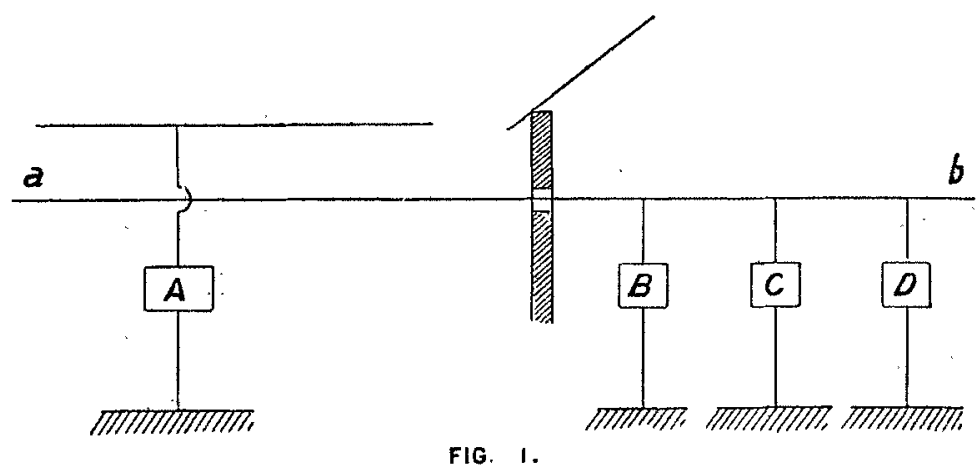

L'installation idéale de ce genre cst représentée dans la figure 2. Dans celle-ci, A représente une ligne de terre en acier montée au-dessus de la ligne et destinée principalement ì İa protéger contre les coups de foudre directs ; B, une batterie de condensateurs branchée en deçà d'une self-induction en fil de fer B', le condensateur combiné à la self-induction ayant pour rôle d'étaler les fronts d'onde trop raides et d'an-

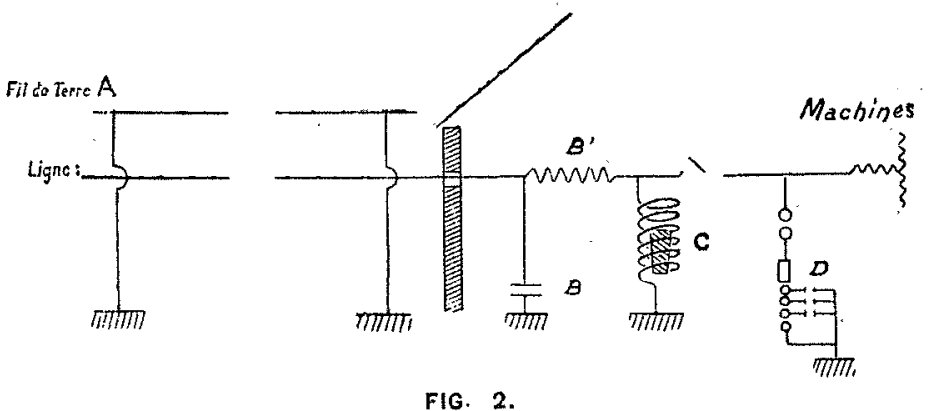

nihiler les effets destrucleurs des phénomènes à haute fréquence ; $\mathrm{C}$, une bobine d'induction à noyau de fer offrant un passage facile aux charges statiques des lignes pour les écouler à la terre; D, une soupape Giles, appareil à très fort débit, destinée à écouler les surtensions de moyenne et de basse fréquence, et par conséquent celles d'origine interne au réseau.

Nous dirons quelques mots sur chacun de ces appareils, ct nous" expliquerons, aussi simplement que possible, leur fonctionnement.

(1) Conférence faite le 23 mars 1912 à la Societé Belge d'Electriciens par M. Carart, sous-directeur de la Socièté générale des Condensateurs électriques de Fribourg.

(2) La Technique moderne, octobre, novembre et décembre 1911 ,janvier février et mars igi2. 


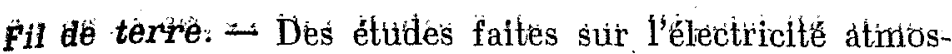

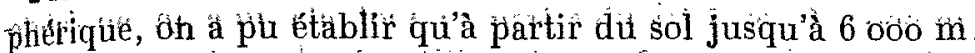

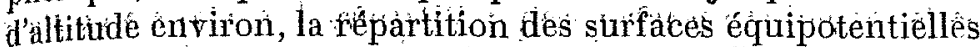

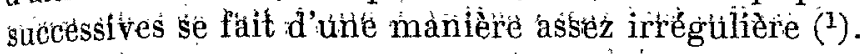

Alors que, par terhṕs serein ét è plainè; là vâleưr du po-

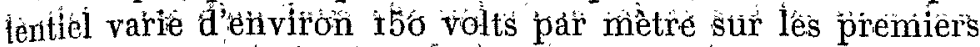
roo m., elle n'est plins que de 40 volts par mètre environ vers les 1 ooo $\mathrm{m}$. et de is vólts par mètre vers les 4 ơóo $\mathrm{m}$. Ces valeirirs n'ont rieh d'absolu, comme on lè sait, et varient même beadicởip áu côturs de la journểe.

La forthatión d'électricicité atmos phếriqute est, en effet, un pliériomene du au fróttenent dê la vapeur d'eau dans l'atnosphere bì d'autres borps ên suspen dans l'atmosphèré. (Expérience d'Armstrong, 1842 : un jet dè vapeưr sortànt d'une chaudière engènare de l'électícité, là chaudiêre s'élec-

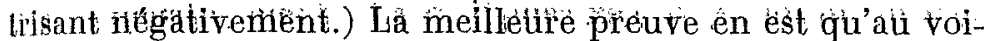
sinage des chutês d'eau les phếnónènés státicưues dảns les lignes piésentent tì cảactêre particuliêrement áigu $\left({ }^{2}\right)$.

Une carisè fréquétête dê troúbles dans les lignes troute son otigune dáns la variátión diurine de l'électricité atmo-

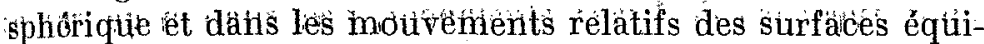
potentielles, s'observant principalement au mornént du levèr et du couchèr du solèil (Looomís; ì 865) (3).

Fail important à tibtèr également; çest qửe la répartition dii ôhamp dans l'ätmósphêre s'écartè cơniplètement des válnùrs indiquées préêdemment au cours d'un oráge. Dans be cas, ơn a pu mesturer $=6600$ à +60 bo volts par mètre

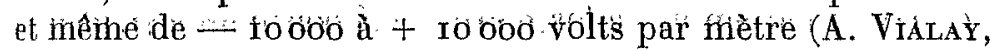
IgIr, op. cit.).

On comprendra àisément l'importance que peut préndre le phếnomene prếcédént aú point de vue des appliciotions des transports d'energie par lignes dériennés. Comme on le sait, ces dernières doiviént souvent passer à dés altitudés considérables pour redéscendre énsuite en plaine, êlles doivent sotivent traverser des contrées très étenduès, de telle sortè qu'ellês cbùpent parfbis dés surfaces équipotentielles a dès potêntiels très differrents, cé qui peut occusionnèn dès phénonomènes statiques dangerètix (4).

Une autre cause de troubles encore dans les lignes, c'est

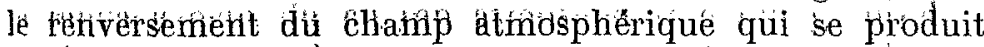

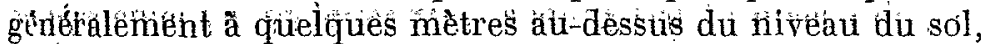
de 4 in. a is tn.; disènt lês météónolơgistes. On vid immédiatemert les inconvénients quiu peưvent résulter de ce phé-

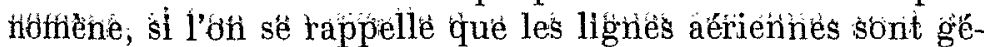
nểralement têndues au-desstis diu sol à des hauteurs tombant dans ces mêthies linites; de telle sorte qu'elles peivènt

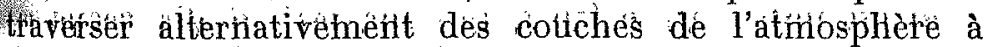
dès potentiels de signine diffếrent.

Ceci posé, on se rendra très bien compte maintenant dia

(1) A. Viatar; Les circulations átmosp̆hériques, Iğr ; A: Gocket, Die Luftelektrizitat, 1908; MACHE et voN SCHWEIDLER, IgI1.

Voir aussi les études de Simipson, 1909 ; Le Cadet, 1904; Chauveau,

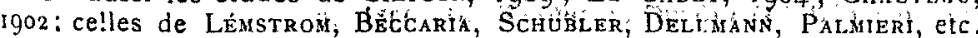

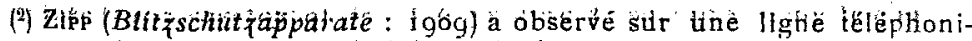
que, coupée à ses deux extrémitésí qu'il était possible d'en tirer deśétin. cêlles très fortes sous infifuence des chárges statiques. Celles ci extaient

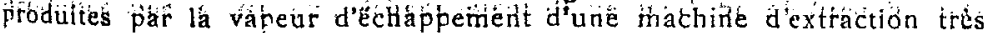
puissante qui montait dans l'atmosphère autour des fils.

(3) Nous connaissons des centrales de la Suisse où en été les interrupteiirs âtitónthatiqués déclenchetit brusquément un peu après le couchér du soleil lorsqu'ils desservent des lignes de montagne, comme s'il se froduisait un véritable court-circuit à l'une des extrémités du conducteur:

(1) On pourra s'en convaincre en lisant, par exemple, les déscriptions

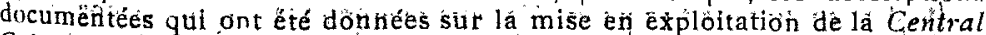
Colorado Pónér. Go ehtré Glenwôd èt Denter (A mérique du Nord). Elle gnt été publiees dans l'Electrical World (1g1o et 1911) et dans les Proceédings of the American Institite of Electrical Engineers (igli). rôle d'un fil de têrie courant au-dessus d'une ligne aérienne. Si l'on se reportè à la figure 3 , sui laquelle nous avons représenté cómment sè répartissent les surfaces équipotentielles au-dessus et au-dessous d'une ligne aérienne, on voit que l'application d'un fil de terre a pour effet de créei autout des conducteùrs sous courant une zone à potentiel zéró. De cétte fáçon, les décharges de la foudre sont dëtournées naturellement à la terre sans emprunter le chemin de la lignè active.

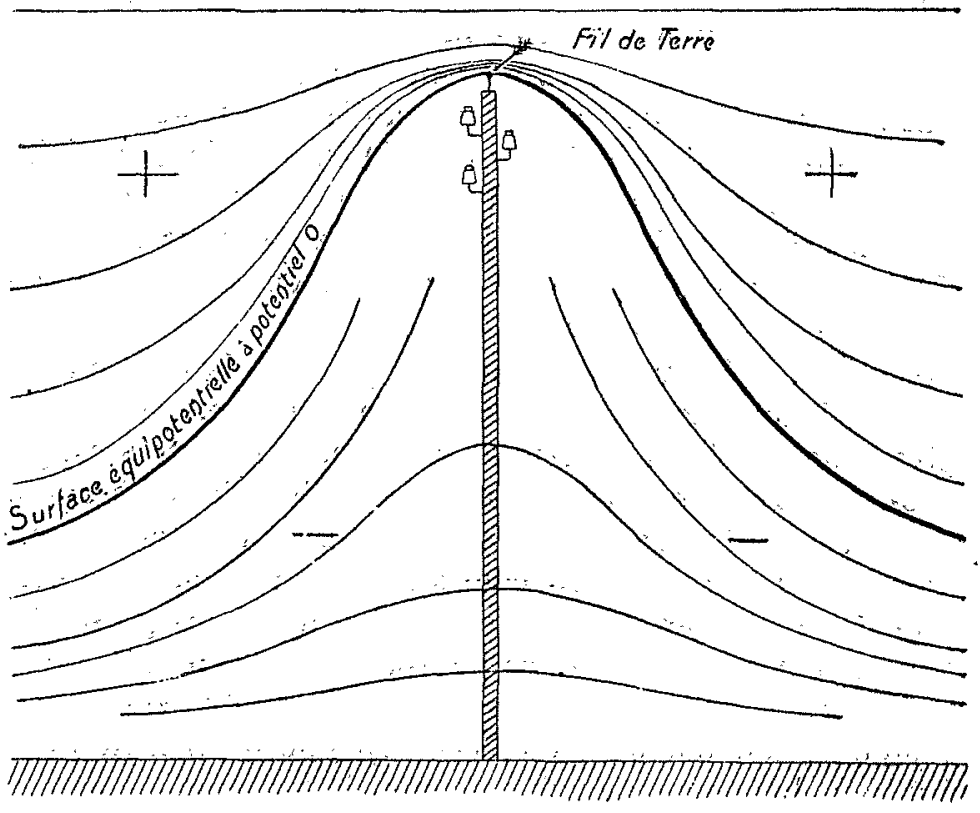

FiG. 3.

La ligne de terrie présentè également ún sérieúx avantage dú fait qu'èlle díninue l'importance des charges statiqués des lignes, dáns lés parcours très accidentés.

En effet, les appareils placés aux extrémités des lignes pour l'écoulcment des charges statiques sont surtout destinés à la protectión efficace des enroulements des machincs et séront souvent sans effet pour la protection de la ligrie elle-même : il suffit simplement que la chàgé électriqué, induite par un nuage éléctrisé, pasśe à une certaine distanc? des extrémités de la ligne et y réste en suspéns (fig: 4); pour que cetto protection né soit plus complètemient ässurée.

Ceci montre lérêl qu'il y a ports d'énergic

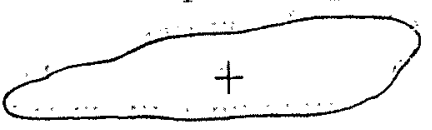
également l'índaris les transde très grande longueur; non protégés par unc ligne de teríe à prévoir

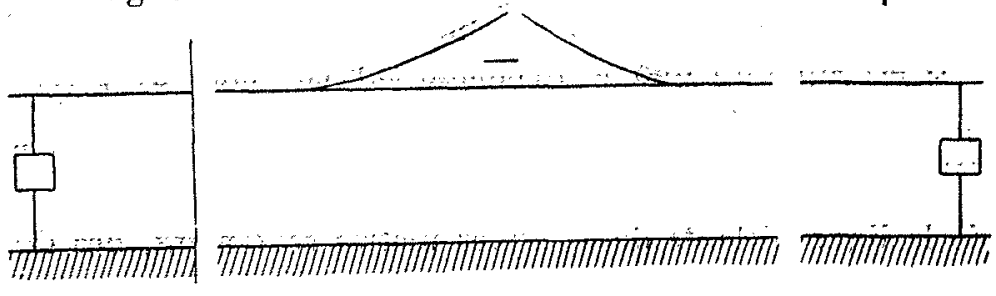

FIG. 4.

des cocfficients de sécurité élevés pour les isolateurs. Gomme je l'ai déjà dit par ailleurs $\left(^{1}\right)$, les conditions ì réaliser pour l'établissement d'une ligne de terre devront être les suivantes:

$I^{\circ}$ Elle devra avoir une résistance élevée au point de vue mécanique, car sa rupture provoquerai: un court-circuit direct au moment où elle entrerait en contact avec la ligne

Technique moderne, t. IV, igra; no $6, p_{213}$ 
active : c'est pourquoi on adopte généralement un câble triessé avec âme en chanvre plutôt qu'un simple fil ;

$2^{\circ}$ Le métal employé sera de l'acier doux galvanisé : on obtient de la sorte un meilleur amortissement des phénomènes inductifs à haute fréquence, dont nous parlerons plus loin, et il résiste mieux aussi aux effets de l'oxydation ;

$3^{\circ}$ Il conviendra, enfin, de la relier en différents points du sol en constituant de bonnes terres.

En fait, si l'application d'un fil de terre au-dessus de la ligne augmente les frais d'établissement de cette ligne dans une certaine proportion, il ne faut pas oublier que, dans les réseaux de très grande importance, il y a un intérêt évident à augmenter de toutes les manières possibles la sécurité de l'installation et à diminuer l'amplitude des phénomènes inductifs dans les conducteurs sous courant, ct d'améliorer les communications téléphoniques lorsque les lignes téléphoniques sont montées sur les mêmes poteaux que ceux du transport de force.

Il est prouvé également que les couches équipotentiellcs nlmosphériques se ferment parfois, au moment des orages, suivant des surfaces sphériques ct, si elles sont très rapprochées, on arrive naturellement à la notion de "l'orage un boule ", ce qui peut être considéré comme un cas limite.

Sans aller jusque-là, on peut s'expliquer de la sorte comment des machines électriques placées dans des postes traversés par des couches de ce genre, en mouvement, ont pu être claquées. Ce phénomìne est d'autant plus subtil qu'il se produit souvent sans èlre accompagné de manifestation visible de la foudre : éclair ou coup de tonnerre.

Nous connaissons une installation, notamment, où deux alternateurs, marchant en parallèle, ont claqué de la sorte, en même temps, et tous les deux à la quatrième bobine $\grave{a}$ partir de la borne d'entrée. C'est pourquoi il est indiqué de prévoir, dans la construction des postes ct des centrales, dés réseaux métalliques extérieurs, comme l'a proposé Semenza, agissant en quelque sorte comme des cages de Faraday, et qui protégeront efficacement les machines contre ce genre dè phénomène.

Condensateurs. - J'ai dil tout à l'heure que le condensateur', branché à l'extrémité d'une Jigne aéricnne, avait pour but d'empêches l'introduclion des ondes ì front très raide ou $\therefore$ hante fréfuence dans les enroulements des machines.

Les prèmières proviennent de ce que le régime du réseau ćtant brusquement troublé en l'un de ses points, un nouveau régime tend à s'établir dans toute son étenduc (Giles, I912). L'onde ayant son origine au point de perturbation se propage de proche en proche dans la ligne.

Il est superllu que je m'étende sur ce genre de phénomène, M. Giles l'ayant dévelóppé récemment à l'Association des Ingénieurs électricions sortis de Montefiore ( ${ }^{2}$ ). Qu'ii me suffise de rappeler que ces ondes créent, entre des points de la ligne distants de quelques mètres seulement, des différences de potenticl assez grandes pour arriver ì faire claquer les enroulements des transformateurs et des machines, non de spires à masse, mais de spire à spire.

Les ondes à haute fréquence d'origine atmosphérique, ongendrées par les décharges inductives, sont à courte période e! produisent naturellement, dans les enroulements, un effet identique à celui qui est provoqué par la propagation des ondes ì front raide. Ft cela s'explique tout naburellement,

(1) GrLes, Bulletin de l'Association des Ingénieurs sortis de Montefore. puisqu'un maximum et un minimum de l'onde à haute fréquence ne peuvent êtrc séparés que par une faible épaisseur de coton de deux spires voisines. On voit de la sorte qu'il est possible de faire claquer l'enroulement d'un transformateur avec quelques centaines de volts, alors que l'appareil lui-même est construit parfois pour plusieurs milliers de volts, 30 ooo ou 40 .000 volts par exemple.

Le condensateur branché à l'entrée des enroulements agịt de manière à étaler le front d'onde, s'il est trop raide, on a absorber l'onde et ì la renvoyer sur la ligne si le phéno. mène est à haute fréquence; dans ce dernier cas, son action est d'autant plus énergique et efficace que sa résistance diminue, comme on le sait, d'une manière inversement proportionnelle à la fréquence du phénomène.

I ies considérations précédentes montrent que le condensateur branché à l'extrémité d'une ligne aérienne agit comme une soupape ou une cloche d'air placée à l'extrémité d'une conduite soumise à des coups de bélier ou à des moúvements vibratoires de la colonne liquide. Elles montrent aussi que des appareils à coupures simples ou multiples, on constituées par de fortes résistances, présenteront de graves inconvénients.

Ils n'entreront en jeu que -lorsque le potentiel ạura déjà acquis une valeur dangereuse dans la partio de l'installation à protéger. Comme l'a fait remarquer très judicieusement Creighton (1), Ic retard que met le parafoudre à comes pour? s'amorcer est assez considérable pour que l'action de l'appireil de protection ne se fasse sentir efficacement que lorsque presque toute la surtension est passéc. De ce fait, si la cone arrive quand même ì s'amorcer, elle n'atura pu agir que comme indicateur de, surtension ct le courant qu'elle écorlera à la tcrre ne sera pas celui de la surtension, comme on le dit à tort, mais bien celui des machines.

Au surplus, j'insiste sur les mots": si elle arrive quand même à s'amorcer, parce qu'il est évident qu'elle ne pourr' s'amorcer par les phénomènes à haute fréquence el ì bassc tension, dangereux comme nous l'avons dit, source certaine d'un très grand nombre d'accidents dans les postes de transformation.

Enfin, comme la résistance des parafoudres à coupures ust généralement très grande, l'efficacité de ces.appareils est réduitc d'autant. Donc, d'une part; si on la fixe à une valeur trop faible, 3oo ohms par exemple, elle donne lieu à dès perturbations dangereuses au moment de leur fonctionnement; d'autre part; si clle est trop forte, de 2 ooo ohms par exemple; la réduction de la valeur absolue de la surtension est insignifiante (Io à 15 pour Ioo) et.l'efficacité du parafoudre, comme nous le disions tout à l'heure, devient illusoire.

A la suite d'expériences inléressantes ( $\left.{ }^{2}\right)$, Steinmetz et Creighton (3) sont arrivés à la conclusion rue l'application des parafoudres à cornes dans les installation de transports d'énergie était une source véritable de danger (Rapport de Creighton au Congrès de Turin, $\mathrm{r}_{9 \mathrm{r}} \mathrm{r}$ ).

D'autres encore sont arrivés à faire la même constatation. Nicholson, par exemple $\left(^{4}\right)$, a donné les résultats comparatifs d'une série d'observations faites sur la ligne Niagara-

(1) Proceedings of the American Institute of Electrical Engineers, mars 1913, p. 579 .

(7) Proceedings of the American Institute of Electrical Engineers, mars I $91 \mathrm{I}$, P. $24 \mathrm{I}$.

(3) Voir egalement Prtrersen, Hochspannungtechnik, i9i I, p. i63.

(1) Proceedings of the American listitute of Electrieal Engineers, sgio p. 24 ". 
Syracuse, de 3 a 4 kms, 60 ono volls, 5o périodes par seconde, sur Ir o7 8 isolateurs, pendant trois années successives. Nous les donnons à notre tour sous forme de tableau :

\begin{tabular}{|c|c|c|c|}
\hline OBSERYATVONS & $\begin{array}{l}1907 \\
1 \text { rotertion par } \\
\text { T50 cornes }\end{array}$ & $\begin{array}{l}1008 \\
\text { Protection pir } \\
\text { Tit0 cornes }\end{array}$ & $\begin{array}{c}1909 \\
\text { Eupuresion } \\
\text { des corups }\end{array}$ \\
\hline Isolateurs çaqués... & 59 & 139 & 1 \\
\hline $\begin{array}{l}\text { Isolateurs endommagés, mais ne de } \\
\text { mandant pas à être remplace } \\
\text { immédiatement............. }\end{array}$ & $\begin{array}{l}\text { e- } \\
\text { es } \\
\text {. } \\
16\end{array}$ & 35 & 13 \\
\hline $\begin{array}{l}\text { Interruptions de service par claquag } \\
\text { d'isalateurs entrainant un arrê } \\
\text { durable dans l'exp!oitation.... }\end{array}$ & $\begin{array}{l}\text { ge } \\
\hat{e} t \\
. \quad 12\end{array}$ & 20 & 1 \\
\hline Interruptions assez courtes dan & & & \\
\hline $\begin{array}{l}\text { l'explottation } \ldots \ldots \ldots \ldots \ldots \ldots \\
\text { Jours v'orage. } \ldots \ldots\end{array}$ & . $\quad 32$ & 38 & 19 \\
\hline
\end{tabular}

On peut voir, par col exemple, que la suppression puro a simple des cornes dans cetle instatlation avait conduil déja ì anc diminulion considérable des claquages d'isolatours ef des interruptions de service.

Mais, diat-on, lapplication jualique des condensalcurs répont-elle entièrement aux conceptions théoriques qui viennent d'être cxposées?

A lapui des explications domées ri-dessus, il nous sefait pussible de remettre sous les yeux du lecleur les rapporls acessivement inléressants qui ont été domnés à l’auleur par diverses exploilations de la Suisse romande, qui appliquent fe:s cowdensateurs dans lcurs réseaux, rapports que nous avous publiés daus La Technique moderne. Comme nous ne roulons pas nous exposer à des rediles, nous citcrons plutot deux aulves excmples, choisis entre plusicurs, le premier concernant une installation en France of le second une inslallalion en Allemagne.

Le directeur de la Sociélé des Forees molrices d'Auvergne nous a écrit en son lemps la letue suggestive suivante:

"Depuis le commencement de l'année g gos, époque à likuelle mous avons procédé à l'installation du matéricl de protection de Fribourg sur notre réseau à 10 ooo volts, it l'est plus survenu ancun accident de transformalcur ou de machine du fail de perturbations almosphériques of de surtensions en ligne.

"Nous sommes lrès heureux d'aroir à vous faire connaître ces heurcux résullats, d'autant plus que les accidents ayaient été nombreux pendant les précédentes ammées et que, d'aulre part, pendant cotte même période, il s'en est produit sur notre réscau à 25000 rolls que nous n'avons pas protégé jusqu’ici par le système des condensateurs."

En ce qui concane le deuxième exemple annoncé, on le frouvcra décrif tout au long dans l'Electrotechnische Zeitschifl du 7 déccmbre זg1 $\mathrm{t}, \mathrm{n}^{\circ}$. 49. Dans unc conférence domnée à Rheydt, à l'Association des Ingénieurs allemands, Jo docteur Frank, jngénieur en chef du réseau de Ncuss, près do Cologne, a déclaré que l'applicalion des condensalours de Fribourg dans son réseau avail fait tomber le nombre des accidenls de 70 pour too de ce qu'il était auparayant, lors de l'utilisation des cornes, malgré les conditions d'exploilation irès difficiles.

Bobines d'induction à noyau de fer pour l'écoulement des charges statiques. - Nous avons insisté précédemment sur limporlance des phémomènes statiques dans les lignes aćíennes. Pour les écouler à la terre, dans les centrales et dans les posles, on dispose de deux moyens assez opposés connur principe : apparéls à jels d'eau ou à colonnes d'cau, on bien bobines de self-induclion à noyau de fer.

Comme on le sait, l'inconvénient du premier de ces appareils est d'avoir constamment le même débit en alternatif cl an conlinu el de conduire par conséquent à uno grande perle d'énergie à la terre, sans compter qu'il n'est pas toujours possible de disposer facilement d'un courant d'eau.

C'est pourquoi, en nous appuyint sur uno série d'expériences, nous préférons préconiser l'emploi de la bobine d'induction à noyau de fer. Ia résistance ohmique de cetle dernière étant Irès faible, les charges statiques s'écouleront facilement à la terre sous forme de courant continu ; le débit en courant alternatif sera au contraire tres faible et la perte d'énergie pourra done être considérée comme insignifianle.

Iâtons-nous d'ajouter que l'application d'apparcils à coupures ne serait pas indiquée non plus en ce cas, l'adoption d'un limiteur $\grave{a}$ fonctionnement continu branché dircetement au réseau étant absolument nécessaire pour l'écoulement de ce genre de phénomène.

Au surplus, il n'est pas mauvais qu'on sache que eertains appareils à coupures peuvent être considérés comme dangereux si on les branche à une ligne aérienno dans laquelle j) y aunit prodnction ćrentudle de charges statiques. Par exemple, Jes apparcils à roulcaux, d'unc part, s'amorcent plus difficilement avec du comant conlinu, ol, d'antre patr, somt tels cue le soullage s'y effectue plas facilement avec le courant alternalif quavec le coumant continu. Nous avons pu obscrver nous-mêmes que sur des appareils Würtz construits pour 15000 volts alternatifs, l'are pouvait cncore être cntretenu avec di courant continu d'environs $x_{7}$ ooo volts. Or, comme les phénomènes statiques s'écoulent à la terre sous forme de courant continu, nous croyons inulile d'insister sur le danger que peuvent présenter ces appareils, s'ils sont branchés à une ligne cxposće à ce genre de phénomènes.

Soupapes Giles. - Nous avons dit tout à l'heure que cet appareil est destiné à écouler les surtensions à moyenne ot à basse fréquence. Celles-ci prennent généralement leur origine dans le réseau même : résonances, fonctionnement d'intermpleurs, claquage de câble, variations brusques de régime, mauvaises mises en parajlèle, etc.

Cos phénomènes sont également assimilables aux coups di bélier dans les conduites hydrauliques, mais ne se produisent pas avec la même soudaineté que lorsqu'ils sont d'origino almosphérique.

En poussant plus loin la comparaison de ce quj se passe dans les conduites hydrauliques et électriques, nous pourrons rappeles que, sur les premières, on place des soupapes et des réservoirs d'air ayant des sections comparables à celle de la conduite elle-même ef " il ne viendrait à l'idée de personne de donner à une soupape une section de $\mathrm{r} \mathrm{dm}^{2}$ quand la conduite elle-mème aurait $r . \mathrm{m}^{2}$ de section. "(Giles.)

Il en cst de même dans les canalisations électriques dans lesquelles il fallait pouvoir arriver à placer des soupapes ayant une résistance ohmique du même ordre de grandeur que l'impédance du réseau.

Encore faul-il réaliser celte condition de telle manière qu'il ne puisse se produire une espèce de coup de marteat en cas de fermelure brusque de la soupape. En intercalant de même, d'un scul coup, une résistance trop faible (comme dans les parafoudres à cornes par exemple) entre deux conducteurs d'une ligne électrique, on engendrera de la sorte une surtension aussi forte que celle qu'il fallait éviter.

Dans la soupape Giles, au contraire, par suite du grand nombre de colonnes en parallele et par suite d'un autre disposilif qui permet le soufllage de l'arc à chaque demi-période, on arrive à n'intercaler cette très faible résistance que progressivement, c'est-à-dire au fur et à mesture de l'augmentation ds la valeur de la surtension " l'appareil fonc- 
tionne donc en quelque sorte comme un rhéostat bien gradué. La pratique a d'ailleurs montré qu'il en était bien ainsi et qu'il était possible de prévoir, s'il le fallait, l'adoption de soupapes pour lesquelles la résistance combinée alteigne seulement 70 ohms.

Enfin, une des caractéristiques de la soupape Giles esti de faciliter l'amorçage des distances explosives d'une même colonne. Fn effet, le nombre d'intervalles d'air de cet appareil cst tel que la surtension d'amorçage scrait considérable s'il n'y avait pas un dispositif spécial prévu à cet effet. Ce dernier consiste à reporter successivement la totalité de la tension du réseau sur chacune des distances explosives, de telle sorte qu'on amorce celles-ci les uncs après les autres au lieu de les amorcer toutes en même temps, comme cela a lieu dans les autres appareils.

Il nous reste à ajouter un mot sur le groupements ou mode de connexion donné habituellement à ces soupapes ; comme les surtensions ì basse et moyenne fréquence peu-

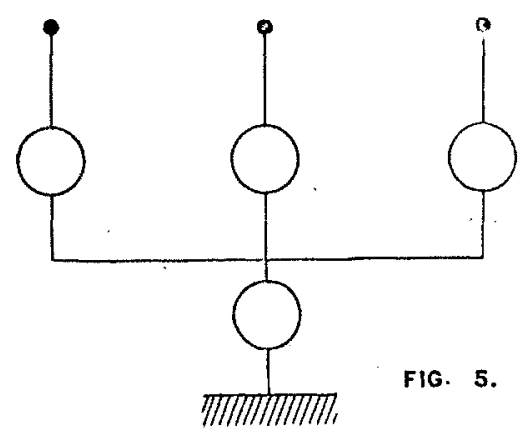
vent sc faire sentir aussi bien entre phases qu'entre chaque phase ret la terre, il sera préférable d'adopter le montage par 4 ide la figure 5. Ainsi qu'on peut le voir, cette disposition a, comme avanlage, d'opposer aux surtensions toujours deux appareils en séric, calculés

pour la moitié de la tension normale, qu'elles se produisent entre conductcurs ou entre chaque conducleur et la terre.

Avant de terminer, je crois devoir dire un mol sur la question de la misc à la terre du point neutre dans les installations à courants triphasés. Cettc question a toujours préoccupé installaleurs ef exploitants el a été, depuis longtemps, le sujet de nombreuses controverses.

Des nombreuses expériences qui ont été failes avec l'un ou l'autre système, et que nous avons pu observer personnellement, nous croyons pouvoir dire, sans être contredil par personnc, que la mise directe du neutre à la terre présente trois sérieux inconvénients :

$r^{\circ}$ Augmentation du danger de mort, au cas où quelqu'un vient ì touchcr des conducleurs sous courant;

$x^{\circ}$ Mise en court-circuit directe sur la machine de la phase qui scrail mise accidentellement ì la terre, ce qui enlève toule possibilité évenluelle de marcher avec les deux autres phases, s'il y a un défaut d'isolement à la troisième;

$3^{\circ}$ Perturbations téléphoniques très forles dans les réseaux de ville à câbles souterrains, ces dernières reudant même l'exploitatión téléphonique impossible si les alternateurs ont des harmoniques de denture d'ordre $3\left({ }^{1}\right)$.

Toutefois, comme la mise du neutre à la tere présculc de nombreux avanlages, nous préconisons de la réaliser par. l'intermédiaire d'une résistance hydraulique, ou par un artifice de ce genre, ce qui supprime, par conséquent, la plupart des inconvénients que nous venons de citer. Il ne nous rst malheureusement pas possible de nous élendre plus longuement sur ce point dans une causerie où l'on ne s'occupe que de la question de la protection des réscaux contre les surtensions.

Bruxelles, le 23 mars 1912.

(1) Voir La Revue électrique, t. XI, 30 awril rgog. r. 304.

\section{SERVICE D'ÉTUDES DES FORGES HYIRAULIOUES DANS LA RÉGION DES PYRÉNÉES}

Nous avons tenu nos lecleurs au courant de l'organisation du Service d'études des grandes Forces hydrauliques, ct des résultats obtenus dans la région des Alpes. Inulile d'en faire, une fois de plus, ressortir toule l'importance, car il n'est persomnc qui, ayant à s'occuper de chutes d'eau, n'ail cu recours aux précieux documents publiés par le Service.

On sait qu'il a été étendu aux cours d'eau des Pyrénées, et prochainement nous nous proposons de donner ici une analyse des travaux ellectués dans cette région. Mais, sains attcndre davantage, nous teinons à signaler la publication qui vient d'être faite en librairie des premiers résultats obtenus au 3 I décembre s 9 ro.

Ils occupent deux tomes des Annales de la Dircction de J'Hydraulique agricole. Le premier, de 260 pages, illustré de superbes vues photographiques, contient les rapporls de M. R. Tavernier, inspecteur général et de M. Matterke ingénieur en chef des Ponts el Chaussées, sur les opérations faites, puis les résultats obtenus pour le bassin de l'Adour. Le tome II est formé de 5 ro pages de barêmes et courbes de jaugeages, plus 62 graphiques de débits des cours d'eạ du bassin de la Garonne.

Nous pensons que ce renscignement sera ulile à plus d'un lecteur, car les Pyrénćes, tout d'abord délaissées pour les Alpes, exportant leur houille blanche par de grandes lignes de transport, semblent par contre devoir etre bienlôt le pays d'ćlection du four ćlectrique, apte à faire mettre en valeur leurs richesses minérales très nombreuses.

\section{LA DURANCE ET SON UTILISATION POUR L'AGRIGULTURE ET L'INDUSTRIE}

Nos lecteurs se rappellent certainement les études publiés ici sur celle question par $M$. Wulherm, ingénieur en chel des Ponts et Chaussées, à Grenoble. Dans les numéros dé février, mars, mai et décembre rgı, l'inlassable et savant ingénieur, à qui ses travaux si jmportants sur le régime des torrents valent une inconlestable autorité en matière d'aménagement des rivières alpestres, nous a donné avec un apergu de l'utilisation actuclle des eaux de la Durance; l'esquisse des grands projets de régularisation dont ce cours d'eau esl susceptible pour le plus grand bien de l'agriculhire en Provence et de l'industric dans la zone de notre graid poit méditerranécn.

" L'ouvre qui a été réalisée, disail oxcellemment M. Wrureu dans les conclusions résumant colte séric darticles, est considérable, mais dle n'est pas lerminéc. Tes irrigations ont pris un lel développenent que l'can de la Durance devient souvent insuffisante, el cependant il y a d: nouveaux besoins qui se manifestent. L'industric de la houille, blanche vient à peine de naîlre et déjà clle réclame une négularisation du régime de la capricieuse rivièrc. Lit même question se pose d'aillcurs plus ou moins pour tous les cours d'eau des Alpes, car partout l'industrie des grandes forces hydrauliques souffre du manque d'cau, surtout pertdant l'hiver. C'est done à juste titre que l'on se préoceupe de remédier à cetle facheuse situation. Malheureusement, if scra lc plus souvent impossible d'arriver à une solution 